\title{
Neuroimaging Studies on Yoga Practice in Healthy Population
}

\author{
Olivia Gosseries* \\ Coma Science Group, GIGA-Consciousness, University of Liege, Belgium
}

Submission: July 24, 2018; Published: September 04, 2018

*Corresponding author: Olivia Gosseries, Coma Science Group, GIGA-Consciousness, University of Liege, Belgium, Email: ogosseries@uliege.be

\section{Introduction}

Yoga comprises various domains of practices but practically we can divide yoga into three categories: poses exercise (asana), breathing (pranayama), and meditation (dhyana). Asana is usually considered as a form of exercise through actions of the body. Breathing-based practice involves purposeful inhalations and exhalations that can be designated at a specific speed and intensity. Breathing exercise can be practiced in a seating, standing or lying position (savasana). Previous studies have shown various mental and physical health benefits related to yoga. For example, studies examining mental health outcomes have reported decreased feeling of anxiety and depression after yoga practice [1]. Cognitive benefits of yoga in healthy subjects have also been demonstrated with improved spatial and verbal memory [2], increased executive functions [3] and better psychometric abilities [4].

Mental health and cognitive benefits of yoga are now well accepted by the community, but the neuronal changes in the brain associated with these benefits mostly remain to be investigated. Changes in behavior are often associated with changes in neuronal activity within the brain. Understanding what can elicit brain modulations that lead to improved cognition can give insight into the development of cognitive interventions in both healthy and clinical populations. In this article, we will review studies on neural changes detected by neuroimaging techniques that occur through yoga practice, which may lead to mental health and overall wellbeing in healthy population. For each section, we will first describe the technique and then report findings related to yoga intervention and practice.

\section{Neuroimaging Techniques and Yoga Findings}

\section{Positron emission tomography (PET)}

PET measures cerebral metabolic rates for glucose or changes in regional cerebral blood flow as an indirect measure of the neural synaptic activity (Figure 1). A PET scan using fluorodeoxyglucose (18F) summates approximately 30 minutes of cerebral glucose metabolism and allows assessment of regional variations. PET is invasive because it involves the injection of positron-emitting radionuclides with short half- lives. As the radioactive compound accumulates in different regions of the brain and positron annihilations occur, the scanner detects the coincident rays produced at all positions outside the head and reconstructs an image that displays the location and concentration of the radioisotope within a plane of the brain. PET is combined with CT imaging to co-register the functional PET image with an anatomic resolution image [5].

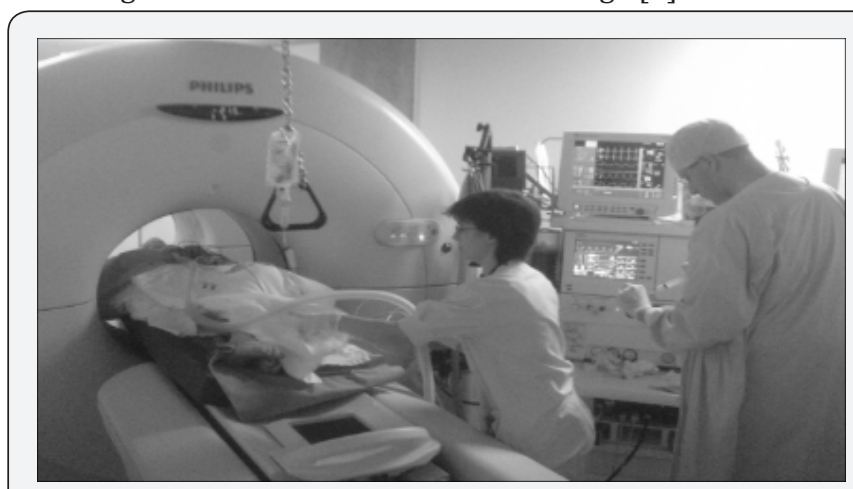

Figure 1: Positron emission tomography (Liege Hospital, Belgium).

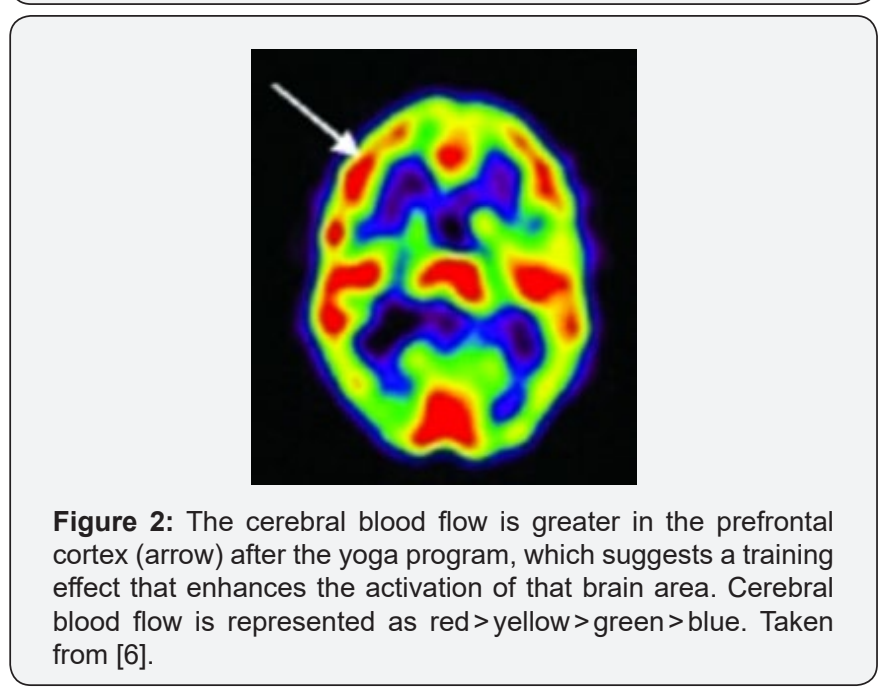

Regarding yoga, decreased blood flow has been measured in the amygdala whereas increased blood flow has been observed in the frontal lobes, after three months of Iyengar yoga training practice in fours subjects [6] (Figure 2). This result suggests that 
yoga training may prevent negative feelings as the amygdala is thought to regulate emotions, and is especially active during negative emotions. Prolonged focus and attention may have been developed throughout the training as the frontal cortex is involved in these processes.

\section{Magnetic resonance imaging (MRI)}

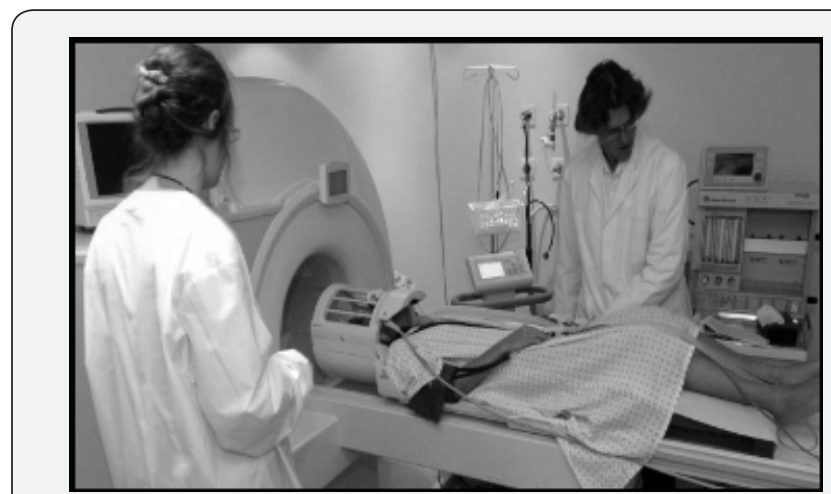

Figure 3: Magnetic resonance imaging (Liege hospital, Belgium).

MRI can detect an increase in blood oxygen concentration that occurs in an area of heightened neuronal activity (Figure 3 ). The basis for this capacity comes from the way neurons make use of oxygen. Functionally induced increases in blood flow are accompanied by alterations in the amount of glucose the brain consumes but not in the amount of oxygen it uses. Additional blood to the brain without a concomitant increase in oxygen consumption leads to a heightened concentration of oxygen in the small veins draining the active neural centers [5]. The reason is that supply has increased, but the demand has not. Therefore, the extra oxygen delivered to the active part of brain simply returns to the general circulation by way of the draining veins. The commonest form of functional MRI
(fMRI) is blood oxygenation level dependent (BOLD) imaging [7]. The BOLD signal depends on the ratio of oxygenated to deoxygenated hemoglobin. In regions of neuronal activity this ratio changes as increased flow of oxygenated blood temporarily surpasses consumption, decreasing the level of paramagnetic deoxyhemoglobin.

These localized changes cause increases in magnetic resonance signal, which are used as markers of functional activation. So far, only a few MRI studies have investigated the benefit of yoga on brain structure and function. Froeliger et al. [8] showed that while viewing negative emotional images and when presented with distracters, seven practitioners of hatha yoga and meditation presented less activation in the dorsolateral prefrontal cortex as compared to seven non-practitioners. This finding suggests that yoga training may decrease negative emotional stimuli from distracting working memory, and thus improve negative emotional response to incoming sensory information. The authors also reported a correlation between amygdala activation and decay of positive affect in nonpractitioners, which was not the case for the yoga trainees. More specifically, non-practitioners showed a decreased positive affect that could be predicted by the magnitude of the activation of the amygdala. Additionally, a positive correlation was found between gray matter volume in the frontal lobe and yoga experience, which suggests that long-term practice of yoga increases gray matter volume. Another study on fourteen yoga practitioners found that asana-based yoga practitioners with six to eleven years of experience (doing four to ten practices a week) also presented increased gray matter, mostly in the insular cortex, area involved in the pain matrix [9]. This increase in gray matter volume correlated with higher pain thresholds during thermal and pain tasks. Gray matter volume in the insula was also increased with yoga experience in this study.

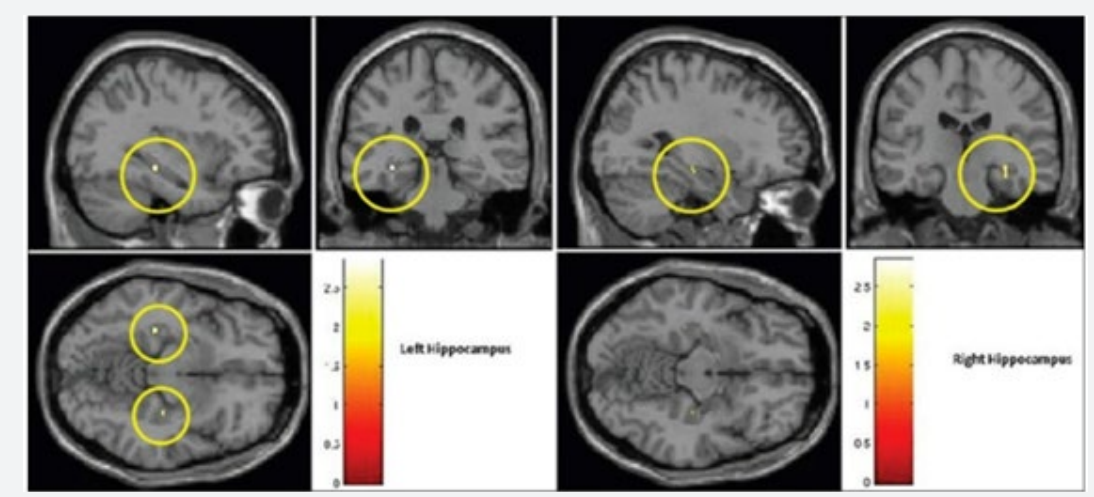

Figure 4: Increased hippocampus gray matter volume after yoga practice in healthy elderly participants (difference before and after training using voxel based morphometric analysis). Taken from [10].

Another study measured the volume of the hippocampus in seven healthy elderly adults before and after an extensive 6-month yoga intervention with asana-based and breathingbased training (including "OM" chanting) [10]. The hippocampus (brain region involved in memory formation and vulnerable to loss of grey matter with aging) was found to increase substantially after the yoga intervention. The increase in gray matter was observed in bilateral hippocampus (Figure 4), which suggests that yoga may have the potential to reduce neuronal senescence. Note, that the sample size of this study (as well as all the studies mentioned so far) is small and there is no control group for comparison, so the results should be taken with caution. However, the increase of gray matter volume was recently confirmed in the left hippocampus of 13 other experienced 
yoga practitioners, compared to 13 controls [11]. The authors also found less activation in the dorsolateral prefrontal cortex in these yoga practitioners during a working memory task (compared to controls), which may indicate increased efficiency [11]. Similarly, increased cortical thickness was measured in the left prefrontal region of 21 older woman practicing hatha yoga for more than 8 years compared to 21 women naive to yoga [12], as previously observed with PET [6].

Finally, in another fMRI study of 12 subjects (four with formal training in yoga including meditation, and eight naïve), the audible "OM" chanting, which produces a sensation of vibration, induced in both groups deactivation in the amygdala, anterior cingulate gyrus, hippocampus, insula, orbitofrontal cortex, parahippocampal gyrus and thalamus, when compared to the brain at rest [13] (Figure 5). These brain regions are part of the limbic system, which controls emotions and drives. In contrast, deactivation did not occur in these brain regions during a control condition (i.e., enunciating "sss", which does not have the vibratory property). This study shows the direct effect of "OM" chanting on brain modulation. But this is however not strictly related to yoga practice as naïve subjects were included, and it rather shows the brain experience of audible "OM".
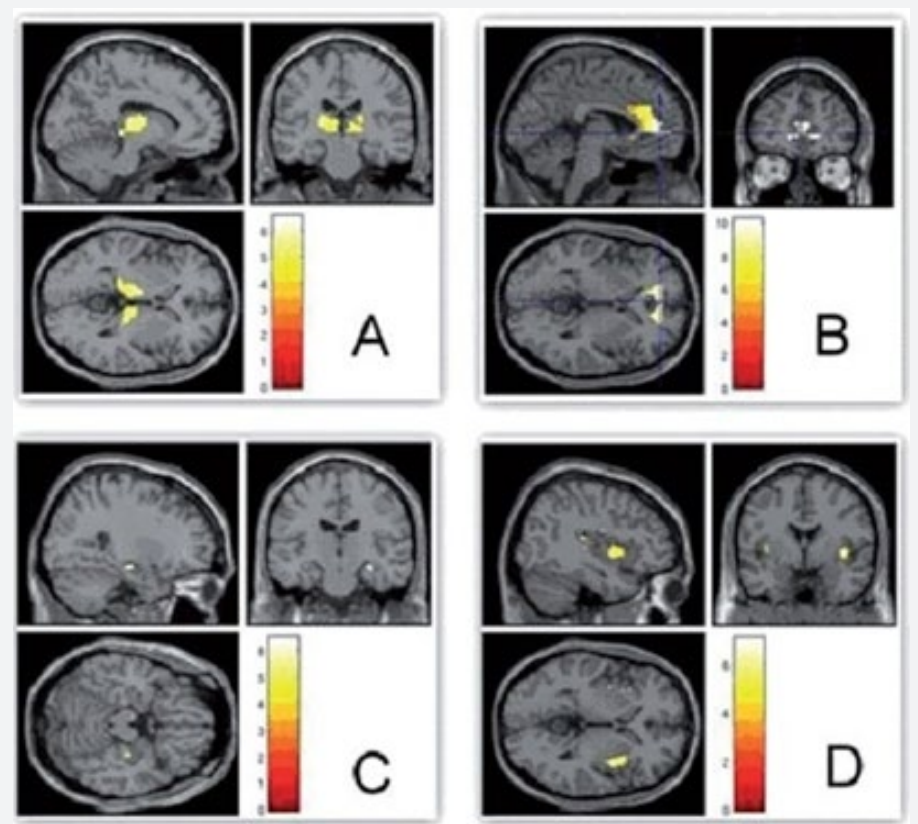

Figure 5: Compared to resting state, "OM" chanting produced deactivation of thalami $(\mathrm{A})$ and limbic structures such as anterior cingulum (B), hippocampi (C) and insula (D). Taken from [13].

\section{Electroencephalography (EEG)}

EEG is a non-invasive technique that allows the detection of spontaneous brain electrical activity from the scalp. It provides temporal resolution in the millisecond range. Traditional EEG technology provides insufficient spatial detail to identify relationships between brain electrical events and structures and functions visualized by fMRI. Recent advances help to overcome this problem by recording EEG from more electrodes, by registering EEG data with anatomical images, such as structural MRI, and by reducing the distortion caused by volume conduction of EEG signals through the skull and scalp. In addition, statistical measurements of sub-second interdependences between EEG time-series recorded from different locations can help to generate hypotheses about the instantaneous functional networks that form between different cortical regions during mental processing [5].

Desai \& colleagues [14] recently reviewed the current literature on EEG and yoga. Based on 15 articles, they concluded that yoga increased overall brain electrical activity, which include increases in alpha, beta and gamma band activity. Alpha band neural oscillations are in the frequency range of $8-12 \mathrm{~Hz}$ and are predominant in the occipital brain areas during periods of eyes closed. In a study from 1992 on eight healthy subjects, an increase in alpha activity was measured over 30 consecutive days of breathing and relaxation yoga training of 50 minutes [15]. More specifically, increases in alpha wave activity were observed in occipital and prefrontal cortices. Another study reported increased alpha band and beta band $(12-30 \mathrm{~Hz})$ activation during pranayama practice (i.e., alternate nostril breathing) in eight long-term practitioners [16]. On the other hand, a recent study that looked at the effects of yoga asana training, pranayama training and a combination of both on brain activity in 80 healthy subjects, found a significant increase in alpha wave activity in asana group and in the combination group, but not in the pranayama group [16]. Another study reported that Kriya yoga and pranayama yoga practitioners presented larger beta waves and spurts of alpha waves (compared to controls), indicating relaxation with the co-occurrence of alertness [18]. Kriya-based 
yoga practice in eight subjects has also been shown to elicit theta activity (slower activity than alpha and beta), with an increase of $40 \%$, mostly in parietal regions [15]. This finding suggests that the brain was more deeply relaxed after a session of yoga with reduced feelings of anxiety and better emotional control. Additionally, the effects on gamma $(>35 \mathrm{~Hz})$ and beta $(15-35 \mathrm{~Hz})$ wave activity were also investigated after a pranayama practice (i.e., producing a vibrating sound while exhaling through the nose) and showed again an increase in both frequency bands during pranayama in eight healthy subjects [19].

\section{Yoga Research, What is Next?}

In summary, the results from the reviewed articles were all in favor of yoga practice. Among yoga practitioners and after a yoga intervention, the three subdivisions of yoga (i.e., asana, meditation and breathing) produced changes in behavior, in cognition and in the brain. Indeed, yoga training has been correlated with decreased amygdala activation and decreased negative emotion in response to emotional distracter images. Yoga also seems to have a constructive effect on the anatomy of the brain, with an increase of grey matter in the hippocampus and frontal regions in elderly subjects. It also seems to have positive effects on brainwave activity in terms of increasing alpha, beta, and theta electrical activity, which have been associated with improvements in cognition, memory, mood, and anxiety.

We are still however at the beginning of yoga research and most findings come from small sample size, uncontrolled and non-randomized studies. Comparing different groups are crucial for accurate testing and interpretation of yoga intervention experiments. Selecting appropriate comparison groups can however be difficult because yoga includes a heterogeneous set of practices. To isolate the specific mechanisms of yoga's effects, using an active control group seems to be the best option, for example a group doing physical exercise other than yoga [20]. Yoga intervention is another good option and in an ideal world, one wants to combine both: group comparison (between experienced and naïve subjects) and yoga intervention (before and after, within subject). Further research should examine the effects of yoga training into the clinical treatment of neurological and psychosocial disorders [20]. Because of the extensive variety of yoga practices, more studies should also directly compare the different styles of yoga and evaluate the extent of their neurocognitive and neuro-anatomical properties on healthy and pathological populations.

\section{Acknowledgement}

This article was supported by the University and University Hospital of Liege, the Belgian National Funds for Scientific Research (FRS-FNRS), the European Union's Horizon 2020 Framework Programme for Research and Innovation (Grant Agreement No. 785907), Luminous project (EU-H2020-fetopenga686764), the James McDonnell Foundation, Mind Science Foundation, and the BIAL Foundation. OG is post-doctoral researcher at FRS-FNRS. I thank Marit Sathrum and Joan Bliefernincht for introducing me to yoga practice and teaching.

\section{References}

1. Streeter CC, Whitfield TH, Owen L, Rein T, Kaarri SK, et al. (2010) Effects of yoga versus walking on mood, anxiety, and brain GABA levels: a randomized controlled MRS study. J Altern Complement Med 16(11): 1145-1152.

2. Naveen KV, Nagarathna R, Nagendra HR, Telles S (1997) Yoga breathing through a particular nostril increases spatial memory scores without lateralized effects. Psychol Reports 81(2): 555-561.

3. Gothe N, Kramer AF, McAuley E (2014) The effects of an 8-week Hatha yoga intervention on executive function in older adults. J Gerontol A Biol Sci Med Sci 69(9): 1109-1116.

4. Sharma VK, Subramanian SV, Bhavanani SK, Madanmohan AB (2014) Effect of fast and slow pranayama practice on cognitive functions in healthy volunteers. J Clin Diagn Res 8(1): 10-13.

5. Boly M, Gosseries O, Massimini M, Rosanova M (2015) Functional neuroimaging and electrophysiology, In: Laureys S, Gosseries O, Tononi G (Eds.), The Neurology of consciousness, $\left(2^{\text {nd }}\right.$ edn), Elsevier, USA, pp. 31-45.

6. Cohen Debbie L, Wintering N, Tolles V, Townsend RR, Farrar JT, et al. (2009) Cerebral blood flow effects of yoga training: preliminary evaluation of 4 cases.J Altern Complementary Med 15(1): 9-14.

7. Ogawa S, Lee TM, Kay AR, Tank DW (1990) Brain magnetic resonance imaging with contrast dependent on blood oxygenation. Proc Natl Acad Sci U S A 87(24): 9868-9872.

8. Froeliger Brett E, Garland EL, Modlin LA, McClernon FJ (2012) Neurocognitive correlates of the effects of yoga meditation practice on emotion and cognition: a pilot study. Front Integr Neurosci, pp. 6-48.

9. Villemure C, Ceko M, Cotton VA, Bushnell MA (2014) Insular cortex mediates increased pain tolerance in yoga practitioners. Cereb Cortex 24(10): 273.

10. Hariprasad VR, Varambally S, Shivakumar V, Kalmady SV, Venkatasubramanian G, et al. (2013) Yoga increases the volume of the hippocampus in elderly subjects. Indian J Psychiatry 55(suppl3): S394-S396.

11. Gothe NP, Hayes JM, Temali C, Damoiseaux JS (2018) Differences in Brain Structure and Function Among Yoga Practitioners and Controls. Front Integr Neurosci 22(12): 26.

12. Afonso RF, Balardin JB, Lazar S, Sato JR, Igarashi N, et al. (2017) Greater Cortical Thickness in Elderly Female Yoga Practitioners-A CrossSectional Study. Front Aging Neurosci 20(9): 201.

13. Kalyani B, Venkatasubramanian G, Arasappa R, Rao N, Kalmady S, et al. (2013) Neurohemodynamic correlates of 'OM' chanting: A pilot functional magnetic resonance imaging study. Int J Yoga 4(1): 3-6.

14. Desai R, Tailor A, Bhatt T (2015) Effects of yoga on brain waves and structural activation: A review. Complement Ther Clin Pract 21(2): 112-118.

15. Satyanarayana M (1992) Effect of Santhi Kriya on certain psychophysiological parameters: a preliminary study. Indian J Physiol Pharmacol 36(2): 88-92.

16. Stancak A, Michal K (1994) EEG changes during forced alternate nostril breathing. Int J Psychophysiol 18(1): 75-79.

17. Trakroo M, Bhavanani AB, Pal GK, Udupa K, Krishnamurthy N (2013) A comparative study of the effects of asan, pranayama and asanpranayama training on neurological and neuromuscular functions of Pondicherry police trainees. Int J Yoga 6(2): 96-103. 
18. Bhatia M, Kumar A, Kumar N, Pandey RM, Kochupillai V, et al. (2003) Electrophysiologic evaluation of Sudarshan Kriya: an EEG, BAER, P300 study. Indian J Physiol Pharmacol 47(2): 157-163.

19. Vialatte FB, Bakardjian H, Prasad R, Cichocki A (2009) EEG paroxysmal gamma waves Bhramari Pranyama: a yoga breathing technique. Conscious Cogn 18(4): 977-988.
20. Park CL, Groessl E, Maiya M, Sarkin A, Eisen SV, et al. (2014) Comparison groups in yoga research: a systematic review and critical evaluation of the literature. Complement Ther Med 22(5): 920-929.

This work is licensed under Creative Commons Attribution 4.0 License

DOI: $10.19080 / J Y P .2018 .06 .555681$ 